\title{
WHICH IS YOUR DIAGNOSIS?
}

Marcelo Souto Nacif ${ }^{1}$, Alair Augusto Sarmet Moreira Damas dos Santos ${ }^{2}$, Clovis Monteiro de Barros Filho ${ }^{3}$, Luciano Carneiro Sales ${ }^{3}$, Valdênia Pereira de Souza ${ }^{4}$, Antonio Carlos Pires Carvalho ${ }^{5}$

Study developed at Hospital de Clínicas de Niterói (HCN), Niterói, RJ, Brasil. 1. Responsável pela Ressonância Magnética Cardiovascular e Médico Radiologista do Hospital de Clínicas de Niterói (HCN), Niterói, RJ, Doutorando em Radiologia (RM Cardíaca) pela Universidade Federal do Rio de Janeiro (UFRJ), Rio de Janeiro, RJ, Brasil. 2. Coordenador de Imagem e Médico Radiologista do Hospital de Clínicas de Niterói (HCN), Niterói, RJ, Brasil. 3. Médicos Cardiologistas do Hospital de Clínicas de Niterói (HCN), Niterói, RJ, Brasil. 4. Médica da Unidade Coronariana do Hospital de Clínicas de Niterói (HCN), Niterói, RJ, Brasil. 5. Coordenador Adjunto do Curso de Pós-Graduação em Radiologia da Universidade Federal do Rio de Janeiro (UFRJ), Rio de Janeiro, RJ, Estagiário em Ressonância Magnética Cardiovascular do Hospital de Clínicas de Niterói (HCN), Niterói, RJ, Brasil. Endereço para correspondência: Prof. Dr. Marcelo Souto Nacif. Rua Tavares de Macedo, 136, ap. 1503/A, Icaraí. Niterói, RJ, Brasil, 24220-211. E-mail: msnacif@yahoo.com.br

Male, 66-year old patient with diabetes and hypertension, weighting $55 \mathrm{~kg}, 1.72 \mathrm{~m}$ in height, body surface area $(\mathrm{BSA})=1.65$, presented at the emergency service with precordial pain, and was referred to the Department of Radiology and Imaging Diagnosis of Hospital de Clínicas de Niterói to be submitted to pharmacological stress cardiac magnetic resonance imaging (MRI).

Figure 1. ECG-gating acquisition for studying the myocardial perfusion during pharmacological stress.

Figure 2. ECG-gating acquisition for studying left ventricular contraction and relaxation during pharmacological stress by magnetic resonance myocardial tagging.
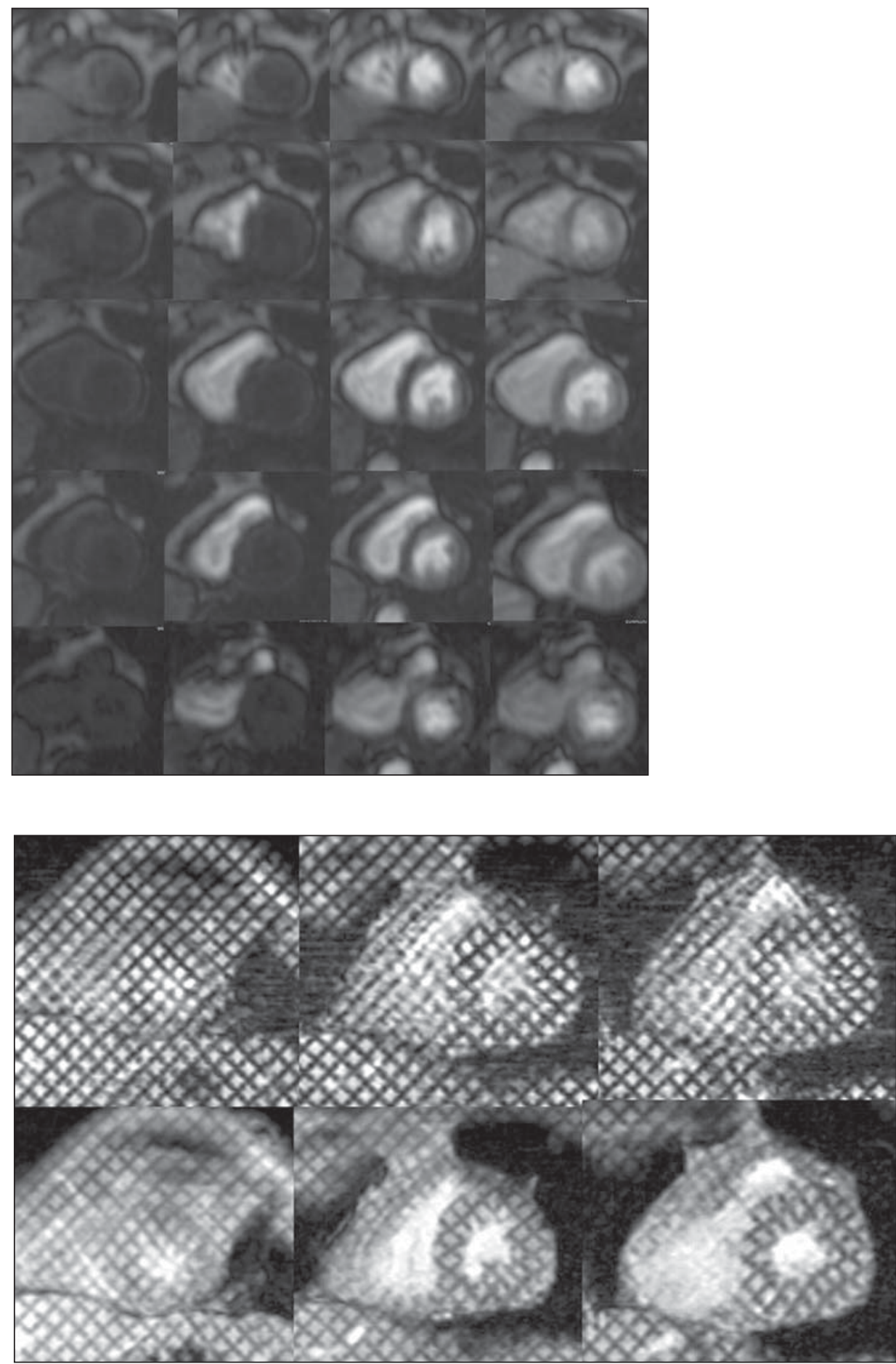


\section{Images description}

Figure 1. ECG-gating acquisition for studying the myocardial perfusion during pharmacological stress. In this case, transmural perfusion deficits can be observed in the anteroseptal, inferoseptal and anteromedial segments, besides nontransmural, subendocardial perfusion deficits in the anterolateral, medial, septal and apical-lateral segments.

Figure 2. ECG-gating acquisition for studying left ventricular contraction and relaxation during pharmacological stress by magnetic resonance myocardial tagging. This aspect demonstrates the presence of hypokinesia in the anteroseptal medial segment, with dipyridamole-induced stress, defining the perfusional deficit severity.

Diagnosis: Myocardial ischemia with anteroseptal medial stress-induced hypokinesia.

\section{COMMENTS}

Myocardial ischemia is a cardiac disorder caused by insufficient blood supply to the myocardium. The decreased blood flow may be due to luminal narrowing of coronary arteries (coronary arteriosclerosis), to obstruction by a thrombus (coronary thrombosis), or, most frequently, to a generalized narrowing of arterioles and other small vessels inside the heart (microcirculation disease). Another factor may be the chronic progression of coronary stenosis with formation of collateral vessels resulting from the slow and persistent reduction in the myocardial blood flow. On the other hand, a prolonged interruption of the blood flow may result in cardiac muscle necrosis (myocardial infarction) ${ }^{(\mathbf{1 , 2})}$.

\section{Myocardial perfusion}

The measurement of myocardial perfusion plays a significant role as guidance for the surgical treatment or hemodynamic intervention in cases of coronary stenosis. Catheterization may accurately define the coronary caliber, but cannot detect the perfusion effect on the cardiac muscle. Stratification of patients should be performed at early stages of the disease, before the symptoms be- come significant, previously to the angiographic study ${ }^{(\mathbf{1}, \mathbf{3})}$.

Scintigraphy represented the first step in the approach of the myocardial perfusion early in the seventies, when the first studies were performed, and, presently, is focused on the study with thallium or sestamibi- ${ }^{99 \mathrm{~m}} \mathrm{Tc}$ single-photon emission tomography (SPECT) and positron-emission tomography (PET). Both these techniques are already recognized as effective in the daily medical practice, however, besides utilizing ionizing radiation and taking 24 hours for the completion of the stress and rest study, these methods present low spatial resolution and high rate of false-positive because of artifacts, when compared with other imaging modalities such as MRI and echocardiography ${ }^{(2,4)}$.

\section{Coronary stenosis effect on the coronary blood flow reserve}

Induction of variable degrees of coronary stenosis in animal models demonstrated that the levels of resting coronary blood flow were maintained until a stenosis with $80-90 \%$ in diameter was achieved. As the stenosis severity increases, the capacity of arteriolar vasodilatation to maintain the flow decreases, and so does the resting coronary blood flow $^{(\mathbf{1}, 4,5)}$

By contrast, the maximum coronary blood flow reserve starts decreasing as the upstream coronary stenosis reaches $50 \%$ in diameter.

There are three levels of resistance influencing the coronary blood flow: the resistance of the large epicardial conductance vessels, the coronary arteriolar resistance, and the subendocardial resistance by parietal tension originating from the ventricular chamber. Under normal conditions, most of this resting resistance is supplied by the coronary arteriolar resistance, and, as the demand increases, most of this increase in the coronary blood flow occurs by means of the decrease in the resistance at this level, potentially increasing up to four times the flow as the demand increases.

Therefore, in a moderately stenosed vessel, the coronary blood flow reserve is decreased, and will be detectable dur- ing the perfusion study with coronary vasodilators $^{(1,5,6)}$.

\section{Hemodynamic effects of stress with vasodilators}

Stimulation with Adenosine $\mathrm{A}_{2 \mathrm{a}}$ receptors on the smooth muscular cells produces vascular relaxation, with maximum arteriolar vasodilation (maximum coronary resistance decrease), allowing the coronary blood flow to reach maximum levels ${ }^{(7,8)}$.

Note: Adenosine has $\mathrm{A}_{1}, \mathrm{~A}_{2 \mathrm{a}}, \mathrm{A}_{2 \mathrm{~b}}$ and $A_{3}$ receptors. $A_{1}$ receptors are present in the sinusal and atrioventricular nodes and mediate the decrease in the heart rate and atrioventricular nodal conduction. $\mathrm{A}_{2 \mathrm{~b}}$ receptors are present in bronchioli and peripheral vasculature, and the stimulation may result in bronchoconstriction and peripheral vasodilation. On the other hand, the $\mathrm{A}_{3}$ receptors are responsible for the preconditioning response $\mathrm{e}^{(\mathbf{7 , 8})}$.

So, after the initiation of an adenosine intravenous infusion, the maximum coronary blood flow is achieved, on average, in 84 seconds, with a variation of up to 125 seconds. Dipyridamole blocks the cellular reuptake of endogenous adenosine and inhibits the adenosine-deaminase responsible for the intracellular adenosine degradation. Therefore, the dipyridamole acts as an indirect coronary vasodilator, increasing the adenosine intracellular and interstitial concentration ${ }^{(4,7,8)}$.

Dipyridamole and adenosine produce vasodilation mediated by both the systemic and coronary adenosine receptor, leading to a mean $8-10 \mathrm{mmHg}$ reduction in the systolic and diastolic arterial pressure, nearly always associated with a reflexive increase in the heart rate. The magnitude of the increase in heart rate is variable, usually ranging between 10 and 20 beats/min. A decrease may be observed in the heart rate response in patients taking beta-blockers, or diabetic patients with underlying autonomic failure ${ }^{(6-8)}$.

\section{Coronary steal}

With dipyridamole or adenosine administration, the resistance vessels in the area supplied by a normal epicardial vessel dilate, reducing the coronary resis- 
tance and resulting in an increment in the blood flow (about five times the normal flow) (myocardial hyperemia). The coronary resistance in a vascular bed supplied by a stenotic epicardial vessel is decreased on rest (coronary reserve already utilized), and little or no vasodilation develops. In other words, the myocardial blood flow in this area does not change or may even slightly decrease because of the peripheral dilation and decrease in the diastolic arterial pressure typical of the pharmacological stress. The result of this process is the coronary steal in the non-stenotic area that remains well or better supplied than the stenotic coronary territory ${ }^{(3,8)}$.

\section{Cardiac magnetic resonance imaging - multimodal}

Cardiac MRI has increasingly been utilized in the clinical practice because of multiple techniques such as cine-MRI, myocardial tagging, perfusion technique, flow study, and delayed enhancement already validated for evaluation of the heart, and mainly, of myocardial ischemia and myocardial infarction (delayed enhancement).

A consensus is still to be reached on the ideal combination of techniques to be utilized for assessment of coronary artery disease, but it is known that accuracy, comfort of the patient, images acquisition time and mainly safety constitute relevant factors to be considered.

Currently, cardiac MRI is performed in our institution according to the following protocol:

I) Pre-examination (5-15 min): Measurement of arterial pressure, heart rate, weight and height. Cardiac monitoring (ECG) and venous puncture at right with a Jelco 18 gauge catheter. Guidance for correct breath hold and positioning during the study. II) First phase (5 min): Imaging for localizing the heart axes. III) Second phase (10-15 min): Dipyridamole is injected for four minutes and, at its peak of action, perfusion imaging is performed during the gadolinium injection and cineMRI for function under stress. IV) Third phase (15-30 $\mathrm{min}$ ): The dipyridamole effect is reversed by aminophylline infusion and cardiac images acquisition is performed in all planes by cine-MRI and aortic and pulmonary flow study. $V$ ) Fourth phase (30-35 min): Rest perfusion images are acquired with a new gadolinium injection. VI) Fifth phase (45$60 \mathrm{~min}$ ): After few minutes, areas of infarction or myocardial fibrosis can be detected with the delayed enhancement technique, complementing the information already obtained.

In this way we could, with a feasible study for clinical investigation, perform a stress examination in at least 45 minutes, and one hour at maximum, by means of a study with high spatial resolution, extremely accurate for different cardiac quantitative and qualitative determinations, with cine-MRI, for example, allow- ing the calculation of left ventricular function, left ventricular mass, and valvular study with the stress and resting perfusion technique (Figure 3), allowing detection of areas of ischemia or reversible or non-reversible perfusional deficit; with the myocardial tagging technique (Figure 4), allowing the segmental analysis of left ventricular contraction and relaxation; and with the delayed enhancement technique, allowing determining the myocardial infarction extent. Some protocols may be performed with angio-MRI for evaluation of the large thoracic vessels, completing the cardiovascular study.

Coronarygraphy (Figure 5) still remains as a standard method in the coronary arteries evaluation, however, coronary MRI

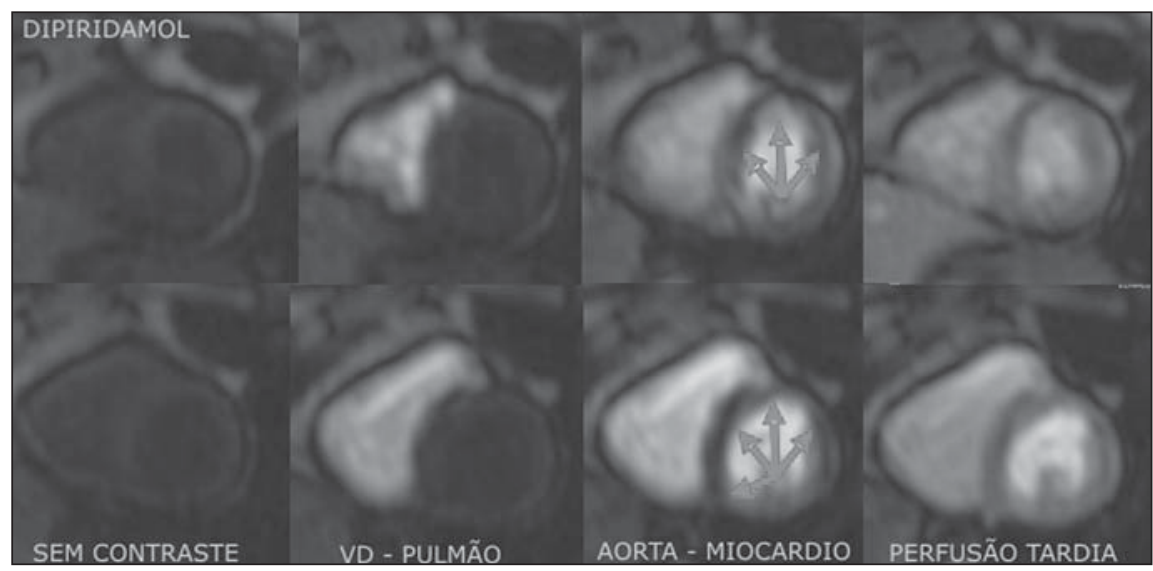

Figure 3. ECG-gated acquisition for evaluation of myocardial perfusion during pharmacological stress. Note the myocardial perfusion deficit (arrows), black images on the normoperfused myocardium (light gray). Note that the gadolinium uptake occurs as usually by the right atrium / right ventricle, and when it passes through the pulmonary artery and returns into the left atrium / left ventricle, delay or signs suggesting right/left shunt or vice-versa cannot be observed. With the filling of the aorta at coronary diastole, we could detect the myocardial perfusion that typically occurs from the endocardium towards the epicardium.

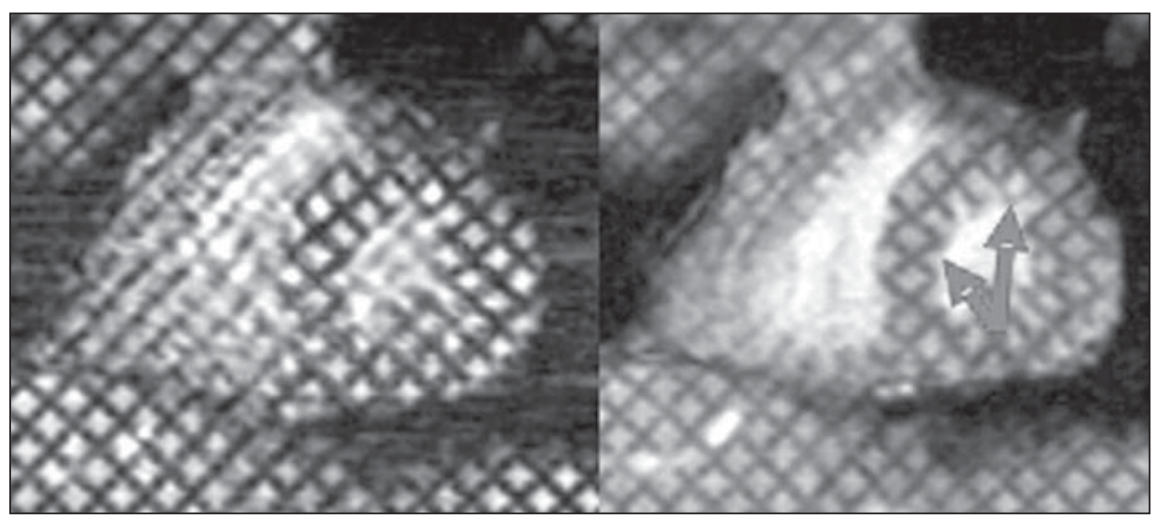

Figure 4. ECG-gated acquisition for evaluation of left ventricular contraction and relaxation during pharmacological stress by the myocardial tagging technique. Note that there is a distortion of the crosshatch pattern on the lateral, inferior and anterior walls, and that, in the septum, more precisely on the medial antero-septal segment (arrows) there is no distortion of the crosshatch pattern. 


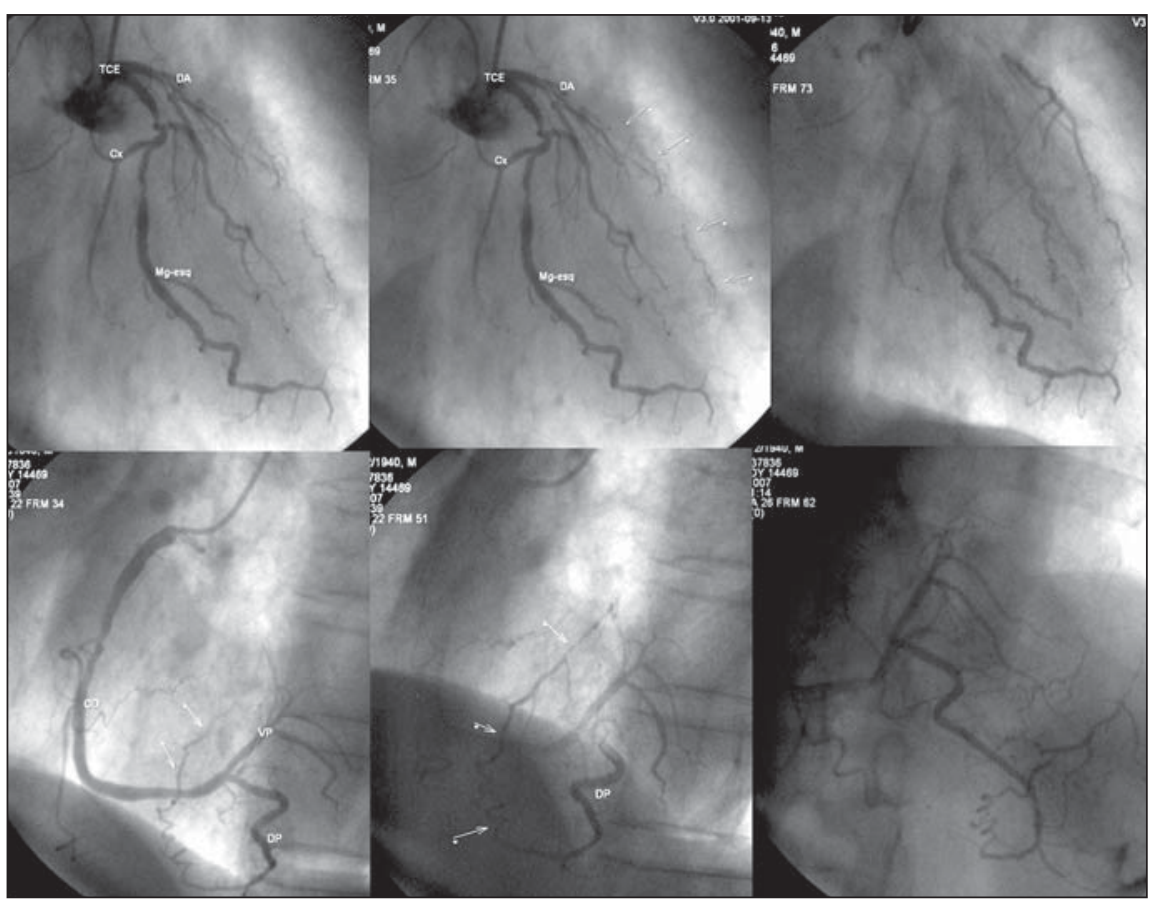

Figure 5. Coronary arteriography - same patient. Coronary artery disease, triarterial lesions. Occlusion of the anterior descending artery (middle third) with distal retrograde filling through the collaterals network originating from the posterior descending artery.

is already a reality in some institutions that may validate the evaluation of atherosclerotic plaques vulnerability, yielding significant information about the coronary artery disease, besides diagnosing luminal reduction.

\section{REFERENCES}

1. Hadlich M, Spoti M, Godoy S, et al. Segurança na realização da ressonância magnética cardíaca pela técnica combinada, utilizando dose máxima de dipiridamol para investigação de isquemia miocárdica. Rev SOCERJ 2007;20:205-211.

2. Nagel E, Klein C, Paetsch I, et al. Magnetic resonance perfusion measurements for the noninvasive detection of coronary artery disease. Circulation 2003;108:432-437.

3. Plein S, Radjenovic A, Ridgway JP, et al. Coronary artery disease: myocardial perfusion MR imaging with sensitivity encoding versus conventional angiography. Radiology 2005;235:423430.

4. Kim RJ, Fieno DS, Parrish TB, et al. Relation ship of MRI delayed contrast enhancement to irreversible injury, infarct age, and contractile function. Circulation 1999;100:1992-2002.

5. Rochitte CE, Kim RJ, Hillenbrand HB, Chen EL, Lima JA. Microvascular integrity and the time course of myocardial sodium accumulation after acute infarction. Circ Res 2000;87:648-655.

6. Rehwald WG, Fieno DS, Chen EL, Kim RJ, Judd RM. Myocardial magnetic resonance imaging contrast agent concentrations after reversible and irreversible ischemic injury. Circulation 2002; 105:224-229.

7. Klem I, Heitner JF, Shah DJ, et al. Improved detection of coronary artery disease by stress perfusion cardiovascular magnetic resonance with the use of delayed enhancement infarction imaging. J Am Coll Cardiol 2006;47:1630-1638.

8. Udelson JE, Dilsizian V, Bonow RO, et al. Cardiologia nuclear. In: Zipes DP, Libby P, Bonow RO, editores. Braunwald - Tratado de doenças cardiovasculares. $7^{\mathrm{a}}$ ed. Rio de Janeiro, RJ: Elsevier, 2006;287-333. 